ITP-95-02E

\title{
Dynamical Chiral Symmetry Breaking by a Magnetic Field in QED
}

\author{
V.P. Gusynin, V.A. Miransky and I.A. Shovkovy \\ Bogolyubov Institute for Theoretical Physics, 252143 Kiev, Ukraine
}

\begin{abstract}
It is shown that the chiral symmetry is spontaneously broken by a constant magnetic field in QED. The dynamical mass of fermions (energy gap in the fermion spectrum) is $m_{d y n} \simeq C \sqrt{e B} \exp \left[-(\pi / \alpha)^{1 / 2}\right]$, where $B$ is the magnetic field, the constant $C$ is of order one and $\alpha=e^{2} / 4 \pi$ is the renormalized coupling constant. Possible applications of this effect are discussed.
\end{abstract}


The dynamics of fermions in an external constant magnetic field in QED was considered by Schwinger long ago [1]. In those classical works, while the interaction with the external field was considered in all orders in the coupling constant, the quantum dynamics was treated perturbatively. There is no dynamical chiral symmetry breaking in QED in this approximation [2]. In this paper we reconsider this problem, treating the QED dynamics nonperturbatively. We will show that a constant magnetic field $B$ leads to dynamical chiral symmetry breaking in massless QED. The dynamical mass of fermions (energy gap in the fermion spectrum) is:

$$
m_{d y n} \simeq C \sqrt{e B} \exp \left[-\left(\frac{\pi}{\alpha}\right)^{1 / 2}\right],
$$

where the constant $C$ is of order one and $\alpha=e^{2} / 4 \pi$ is the renormalized coupling constant relating to the scale $\mu=m_{d y n}$.

The essence of this effect is the dimensional reduction $D \rightarrow D-2(3+1 \rightarrow 1+1$ in this case) in the dynamics of fermion pairing in a magnetic field, pointed out recently in Refs. [3, [1]. Actually, we will show that, in Euclidean space, the equation describing the Nambu-Goldstone (NG) bosons in QED in a magnetic field has the form of a twodimensional Schrödinger equation:

$$
\left(-\Delta+m_{d y n}^{2}+V(\mathbf{r})\right) \Psi(\mathbf{r})=0
$$

Here $\Psi(\mathbf{r})$ is expressed through the Bethe-Salpeter (BS) function of NG bosons, $\Delta=$ $\partial^{2} / \partial x_{3}^{2}+\partial^{2} / \partial x_{4}^{2}$ (the magnetic field is in the $+x_{3}$ direction; $x_{4}=i t$ ), and the potential $V(\mathbf{r})$ is

$$
V(\mathbf{r})=\frac{\alpha}{\pi l^{2}} \exp \left(\frac{r^{2}}{2 l^{2}}\right) E i\left(-\frac{r^{2}}{2 l^{2}}\right), \quad r^{2}=x_{3}^{2}+x_{4}^{2},
$$

where $E i(x)=-\int_{-x}^{\infty} d t \exp (-t) / t$ is the integral exponential function [5] and $l \equiv|e B|^{-1 / 2}$ is the magnetic length.

We emphasize that we work in the conventional, weak coupling, phase of QED. That is, the bare coupling $\alpha^{(0)}$, relating to the scale $\mu=\Lambda$, where $\Lambda$ is an ultraviolet cutoff, is 
assumed to be small, $\alpha^{(0)} \ll 1$. Then, because of infrared freedom in QED, interactions in the theory are weak at all scales and, as a result, the treatment of the nonperturbative dynamics is reliable.

We will consider possible applications of this effect at the end of the paper.

The Lagrangian density of massless QED in a magnetic field is:

$$
\mathcal{L}=-\frac{1}{4} F^{\mu \nu} F_{\mu \nu}+\frac{1}{2}\left[\bar{\psi},\left(i \gamma^{\mu} D_{\mu}\right) \psi\right]
$$

where the covariant derivative $D_{\mu}$ is

$$
D_{\mu}=\partial_{\mu}-i e\left(A_{\mu}^{e x t}+A_{\mu}\right), \quad A_{\mu}^{e x t}=\left(0,-\frac{B}{2} x_{2}, \frac{B}{2} x_{1}, 0\right) .
$$

Besides the Dirac index $(n)$, the fermion field carries an additional, flavor, index $a=$ $1,2, \ldots, N$. Then, at $N \geq 2$, the Lagrangian density (44) is invariant under the chiral $S U_{L}(N) \times S U_{R}(N) \times U_{L+R}(1)$ transformations ( we will not discuss the dynamics related to the anomalous, singlet, current $j_{5 \mu}$ in this paper). Since we consider the weak coupling phase of QED, there is no spontaneous chiral symmetry breaking at $B=0$ [6]. We will show that the magnetic field changes the situation dramatically: at $B \neq 0$ the chiral symmetry $S U_{L}(N) \times S U_{R}(N)$ breaks down to the $S U_{V}(N) \equiv S U_{R+L}(N)$ and, as a result, there appear $N^{2}-1$ gapless NG bosons composed of fermions and antifermions. As we will see, the BS equation for the NG bosons defines the dynamical mass (energy gap) for fermions.

The homogeneous BS equation for $N^{2}-1 \mathrm{NG}$ bound states takes the form (for a review see Ref. [7]):

$$
\begin{aligned}
\chi_{A B}^{\beta}(x, y ; P)= & -i \int d^{4} x_{1} d^{4} y_{1} d^{4} x_{2} d^{4} y_{2} G_{A A_{1}}\left(x, x_{1}\right) K_{A_{1} B_{1} ; A_{2} B_{2}}\left(x_{1} y_{1}, x_{2} y_{2}\right) \\
& \chi_{A_{2} B_{2}}^{\beta}\left(x_{2}, y_{2} ; P\right) G_{B_{1} B}\left(y_{1}, y\right),
\end{aligned}
$$

where the BS wave function $\chi_{A B}^{\beta}(x, y ; P)=\left\langle 0\left|T \psi_{A}(x) \bar{\psi}_{B}(y)\right| P ; \beta\right\rangle, \beta=1,2, \ldots, N^{2}-1$, and the fermion propagator $G_{A B}(x, y)=\left\langle 0\left|T \psi_{A}(x) \bar{\psi}_{B}(y)\right| 0\right\rangle$; the indices $A=(n a)$ and $B=(m b)$ include both Dirac $(n, m)$ and flavor $(a, b)$ indices. Note that though the external field $A_{\mu}^{e x t}$ (司) 
breaks the conventional translation invariance, the total momentum $P$ is a good, conserved, quantum number for neutral channels [8], in particular, for these NG bosons. Since, as will be shown below, the NG bosons are formed in the infrared region, where the QED coupling is weak, one can use the BS kernel in leading order in $\alpha$ [7]:

$$
\begin{aligned}
K_{A_{1} B_{1} ; A_{2} B_{2}}\left(x_{1} y_{1}, x_{2} y_{2}\right) & =-4 \pi i \alpha \delta_{a_{1} a_{2}} \delta_{b_{2} b_{1}} \gamma_{n_{1} n_{2}}^{\mu} \gamma_{m_{2} m_{1}}^{\nu} \mathcal{D}_{\mu \nu}\left(y_{2}-x_{2}\right) \delta\left(x_{1}-x_{2}\right) \delta\left(y_{1}-y_{2}\right) \\
& +4 \pi i \alpha \delta_{a_{1} b_{1}} \delta_{b_{2} a_{2}} \gamma_{n_{1} m_{1}}^{\mu} \gamma_{m_{2} n_{2}}^{\nu} \mathcal{D}_{\mu \nu}\left(x_{1}-x_{2}\right) \delta\left(x_{1}-y_{1}\right) \delta\left(x_{2}-y_{2}\right),
\end{aligned}
$$

where the photon propagator

$$
\mathcal{D}_{\mu \nu}(x)=\frac{-i}{(2 \pi)^{4}} \int d^{4} k e^{i k x}\left(g_{\mu \nu}-\lambda \frac{k_{\mu} k_{\nu}}{k^{2}}\right) \frac{1}{k^{2}}
$$

( $\lambda$ is a gauge parameter). The first term on the right-hand side of Eq.(7) corresponds to the ladder approximation. The second (annihilation) term does not contribute to the BS equation for massless NG bosons (this follows from the fact that, due to the Ward identities for axial currents, the BS equation for the NG bosons can be reduced to the Schwinger-Dyson equation for the fermion propagator where there is no contribution of the annihilation term [7]). Therefore we shall omit this term in the following. Then the BS equation takes the form:

$$
\begin{aligned}
\chi_{A B}^{\beta}(x, y ; P)= & -4 \pi \alpha \int d^{4} x_{1} d^{4} y_{1} S_{A A_{1}}\left(x, x_{1}\right) \delta_{a_{1} a_{2}} \gamma_{n_{1} n_{2}}^{\mu} \chi_{A_{2} B_{2}}^{\beta}\left(x_{1}, y_{1} ; P\right) \\
\cdot & \delta_{b_{2} b_{1}} \gamma_{m_{2} m_{1}}^{\nu} S_{B_{1} B}\left(y_{1}, y\right) \mathcal{D}_{\mu \nu}\left(y_{1}-x_{1}\right),
\end{aligned}
$$

where, since we are working to lowest order in $\alpha$, the full fermion propagator $G_{A B}(x, y)$ is replaced by the propagator $S$ of a free fermion (with the mass $m=m_{d y n}$ ) in a magnetic field [1]:

$$
S_{A B}(x, y)=\delta_{a b} \exp \left(\frac{i e}{2}(x-y)^{\mu} A_{\mu}^{e x t}(x+y)\right) \tilde{S}_{n m}(x-y),
$$

where the Fourier transform of $\tilde{S}$ is

$$
\begin{aligned}
\tilde{S}(k) & =\int_{0}^{\infty} d s \exp \left[i s\left(k_{0}^{2}-k_{3}^{2}-\mathbf{k}_{\perp}^{2} \frac{\tan (e B s)}{e B s}-m_{d y n}^{2}\right)\right] \\
& \cdot\left[\left(k^{0} \gamma^{0}-k^{3} \gamma^{3}+m_{d y n}\right)\left(1+\gamma^{1} \gamma^{2} \tan (e B s)\right)-\mathbf{k}_{\perp} \gamma_{\perp}\left(1+\tan ^{2}(e B s)\right)\right]
\end{aligned}
$$


(here $\left.\mathbf{k}_{\perp}=\left(k_{1}, k_{2}\right), \gamma_{\perp}=\left(\gamma_{1}, \gamma_{2}\right)\right)$. Using the new variables, the center mass coordinate, $R=(x+y) / 2$, and the relative coordinate, $r=x-y$, equation (9) can be rewritten as

$$
\begin{aligned}
\tilde{\chi}_{n m}(R, r ; P)= & -4 \pi \alpha \int d^{4} R_{1} d^{4} r_{1} \tilde{S}_{n n_{1}}\left(R-R_{1}+\frac{r-r_{1}}{2}\right) \gamma_{n_{1} n_{2}}^{\mu} \tilde{\chi}_{n_{2} m_{2}}\left(R_{1}, r_{1} ; P\right) \gamma_{m_{2} m_{1}}^{\nu} \\
& \cdot \tilde{S}_{m_{1} m}\left(\frac{r-r_{1}}{2}-R+R_{1}\right) \mathcal{D}_{\mu \nu}\left(-r_{1}\right) \exp \left[-i e\left(r+r_{1}\right)^{\mu} A_{\mu}^{e x t}\left(R-R_{1}\right)\right] .
\end{aligned}
$$

Here the function $\tilde{\chi}_{n m}(R, r ; P)$ is defined from the equation

$$
\chi_{A B}^{\beta}(x, y ; P)=\left\langle 0\left|T \psi_{A}(x) \bar{\psi}_{B}(y)\right| P ; \beta\right\rangle=\lambda_{a b}^{\beta} \exp \left[i e r^{\mu} A_{\mu}^{e x t}(R)\right] \tilde{\chi}_{n m}(R, r ; P)
$$

where $\lambda^{\beta}$ are $N^{2}-1$ flavor matrices $\left(\operatorname{tr}\left(\lambda^{\beta} \lambda^{\gamma}\right)=2 \delta_{\beta \gamma} ; \beta, \gamma=1,2, \ldots, N^{2}-1\right)$. The important fact is that the effect of translation symmetry breaking by the external field is factorized in the phase factor in Eq.(13) and equation (12) admits a translation invariant solution, $\tilde{\chi}_{n m}(R, r ; P)=\exp (-i P R) \tilde{\chi}_{n m}(r ; P)$. Henceforth we will consider the case with $P \rightarrow 0$. Then, transforming this equation into momentum space, we get:

$$
\begin{aligned}
\tilde{\chi}_{n m}(p)= & -4 \pi \alpha \int \frac{d^{2} q_{\perp} d^{2} R_{\perp} d^{2} k_{\perp} d^{2} k_{\|}}{(2 \pi)^{6}} \exp \left(-i \mathbf{q}_{\perp} \mathbf{R}\right) \tilde{S}_{n n_{1}}\left(p_{\|}, \mathbf{p}_{\perp}+e \mathbf{A}^{e x t}(R)+\frac{\mathbf{q}_{\perp}}{2}\right) \\
\cdot & \gamma_{n_{1} n_{2}}^{\mu} \tilde{\chi}_{n_{2} m_{2}}(k) \gamma_{m_{2} m_{1}}^{\nu} \tilde{S}_{m_{1} m}\left(p_{\|}, \mathbf{p}_{\perp}+e \mathbf{A}^{e x t}(R)-\frac{\mathbf{q}_{\perp}}{2}\right) \\
& \mathcal{D}_{\mu \nu}\left(k_{\|}-p_{\|}, \mathbf{k}_{\perp}-\mathbf{p}_{\perp}-2 e \mathbf{A}^{e x t}(R)\right)
\end{aligned}
$$

where $p_{\|} \equiv\left(p^{0}, p^{3}\right), \mathbf{p}_{\perp} \equiv\left(p^{1}, p^{2}\right)$.

The crucial point for the further analysis will be the assumption that $m_{d y n} \ll \sqrt{|e B|}$ and that the region mostly responsible for generating the mass is the infrared region with $k \lesssim m_{d y n} \ll \sqrt{|e B|}$. As we shall see below, this assumption is self-consistent (see Eq.(代). The assumption allows us to replace the propagator $\tilde{S}_{n m}$ in Eq.(14) by the pole contribution of the lowest Landau level (LLL). In order to show this, we recall that the energy spectrum of fermions with $m=m_{d y n}$ in a magnetic field is [9]

$$
E_{n}\left(p_{3}\right)= \pm \sqrt{m_{d y n}^{2}+2|e B| n+p_{3}^{2}}, \quad n=0,1,2, \ldots
$$


(the Landau levels). The propagator $\tilde{S}(p)$ can be decomposed over the Landau level poles 14, 10]:

$$
\tilde{S}(p)=i \exp \left(-\frac{\mathbf{p}_{\perp}^{2}}{|e B|}\right) \sum_{n=0}^{\infty}(-1)^{n} \frac{D_{n}(e B, p)}{p_{0}^{2}-p_{3}^{2}-m_{d y n}^{2}-2|e B| n}
$$

with

$$
\begin{aligned}
D_{n}(e B, p) & =\left(p^{0} \gamma^{0}-p^{3} \gamma^{3}+m_{d y n}\right)\left[\left(1-i \gamma^{1} \gamma^{2}\right) L_{n}\left(2 \frac{\mathbf{p}_{\perp}^{2}}{|e B|}\right)\right. \\
& \left.-\left(1+i \gamma^{1} \gamma^{2}\right) L_{n-1}\left(2 \frac{\mathbf{p}_{\perp}^{2}}{|e B|}\right)\right]+4\left(p^{1} \gamma^{1}+p^{2} \gamma^{2}\right) L_{n-1}^{1}\left(2 \frac{\mathbf{p}_{\perp}^{2}}{|e B|}\right)
\end{aligned}
$$

where $L_{n}(x)$ are the generalized Laguerre polynomials $\left(L_{n} \equiv L_{n}^{0}\right.$ and $L_{-1}^{\alpha}(x)=0$ by definition). Eq.(16) implies that at $m_{d y n} \ll \sqrt{|e B|}$, the LLL with $n=0$ dominates in the infrared region with $p \lesssim m_{d y n}$.

The LLL pole dominance essentialy simplifies the analysis. Now

$$
\tilde{S}(p) \simeq i \exp \left(-l^{2} \mathbf{p}_{\perp}^{2}\right) \frac{\hat{p}_{\|}+m_{d y n}}{p_{\|}^{2}-m_{d y n}^{2}}\left(1-i \gamma^{1} \gamma^{2}\right)
$$

where $\hat{p}_{\|}=p^{0} \gamma^{0}-p^{3} \gamma^{3}$ and $\hat{p}_{\|}^{2}=\left(p^{0}\right)^{2}-\left(p^{3}\right)^{2}$, and equation (14) transforms into the following one:

$$
\begin{aligned}
& \rho\left(p_{\|}, \mathbf{p}_{\perp}\right)=\frac{2 \alpha l^{2}}{(2 \pi)^{4}} e^{-l^{2} \mathbf{p}_{\perp}^{2}} \int d^{2} A_{\perp} d^{2} k_{\perp} d^{2} k_{\|} e^{-l^{2} \mathbf{A}_{\perp}^{2}}\left(1-i \gamma^{1} \gamma^{2}\right) \gamma^{\mu} \frac{\hat{k}_{\|}+m_{d y n}}{k_{\|}^{2}-m_{d y n}^{2}} \\
& \text {. } \quad \rho\left(k_{\|}, \mathbf{k}_{\perp}\right) \frac{\hat{k}_{\|}+m_{d y n}}{k_{\|}^{2}-m_{d y n}^{2}} \gamma^{\nu}\left(1-i \gamma^{1} \gamma^{2}\right) \mathcal{D}_{\mu \nu}\left(k_{\|}-p_{\|}, \mathbf{k}_{\perp}-\mathbf{A}_{\perp}\right),
\end{aligned}
$$

where

$$
\rho\left(p_{\|}, \mathbf{p}_{\perp}\right)=\left(\hat{p}_{\|}-m_{d y n}\right) \tilde{\chi}(p)\left(\hat{p}_{\|}-m_{d y n}\right) .
$$

Eq.(18) implies that $\rho\left(p_{\|}, \mathbf{p}_{\perp}\right)=\exp \left(-l^{2} \mathbf{p}_{\perp}^{2}\right) \varphi\left(p_{\|}\right)$, where $\varphi\left(p_{\|}\right)$satisfies the equation:

$$
\begin{aligned}
\varphi\left(p_{\|}\right)= & \frac{\pi \alpha}{(2 \pi)^{4}} \int d^{2} k_{\|}\left(1-i \gamma^{1} \gamma^{2}\right) \gamma^{\mu} \frac{\hat{k}_{\|}+m_{d y n}}{k_{\|}^{2}-m_{d y n}^{2}} \varphi\left(k_{\|}\right) \\
& \cdot \frac{\hat{k}_{\|}+m_{d y n}}{k_{\|}^{2}-m_{d y n}^{2}} \gamma^{\nu}\left(1-i \gamma^{1} \gamma^{2}\right) \mathcal{D}_{\mu \nu}^{\|}\left(k_{\|}-p_{\|}\right),
\end{aligned}
$$




$$
\mathcal{D}_{\mu \nu}^{\|}\left(k_{\|}-p_{\|}\right)=\int d^{2} k_{\perp} \exp \left(-\frac{l^{2} \mathbf{k}_{\perp}^{2}}{2}\right) \mathcal{D}_{\mu \nu}\left(k_{\|}-p_{\|}, \mathbf{k}_{\perp}\right) .
$$

Thus the BS equation has been reduced to a two-dimensional integral equation. Of course, this fact reflects the two-dimensional character of the dynamics of the LLL in the infrared region, that can be explicitly read from Eq.(17).

We emphasize that the dimensional reduction in a magnetic field does not affect the dynamics of the center of mass of neutral bound states (in particular, these $\left(N^{2}-1\right) \mathrm{NG}$ bosons). Indeed, the reduction $3+1 \rightarrow 1+1$ in the fermion propagator, in the infrared region, reflects the fact that the motion of charged particles is restricted in directions perpendicular to the magnetic field. Since there is no such a restriction for the motion of the center of mass of neutral particles, their propagator must have a $(3+1)$-dimensional form [11]. This fact was explicitly shown for neutral bound states in the Nambu-Jona-Lasinio model in a magnetic field, in $1 / N_{c}$ expansion [4] and for neutral excitations in nonrelativistic systems [12]. Since, besides that, the propagator of massive fermions is nonsingular at small momenta, we conclude that the infrared dynamics of the NG modes is soft in the present model. This in particular implies that the phenomenon of spontaneous chiral symmetry breaking in this model does not contradict to the Mermin-Wagner-Coleman theorem [13] forbidding the spontaneous breakdown of continuous symmetries at $D=1+1$.

Henceforth we will use Euclidean space with $k_{4}=-i k^{0}$, where the total momentum $P$ of NG bosons equals zero. In order to define the matrix structure of the wave function $\varphi\left(p_{\|}\right)$ of the NG bosons, note that, in a magnetic field, there is the symmetry $S O(2) \times S O(2) \times \mathcal{P}$, where the $S O(2) \times S O(2)$ is connected with rotations in $x_{1}-x_{2}$ and $x_{3}-x_{4}$ planes and $\mathcal{P}$ is the inversion transformation $x_{3} \rightarrow-x_{3}$ (under which a fermion field transforms as $\psi \rightarrow i \gamma_{5} \gamma_{3} \psi$ ). This symmetry implies that the function $\varphi\left(p_{\|}\right)$takes the form:

$$
\varphi\left(p_{\|}\right)=\gamma_{5}\left(A+i \gamma_{1} \gamma_{2} B+\hat{p}_{\|} C+i \gamma_{1} \gamma_{2} \hat{p}_{\|} D\right)
$$

where $\hat{p}_{\|}=p_{3} \gamma_{3}+p_{4} \gamma_{4}$ and $A, B, C$ and $D$ are functions of $p_{\|}^{2}\left(\gamma_{\mu}\right.$ are antihermitian in Euclidean space). 
We begin the analysis of the equation (20) by choosing the Feynman gauge (the general covariant gauge will be considered below). Then,

$$
\mathcal{D}_{\mu \nu}^{\|}\left(k_{\|}-p_{\|}\right)=i \delta_{\mu \nu} \pi \int_{0}^{\infty} \frac{d x \exp \left(-l^{2} x / 2\right)}{\left(k_{\|}-p_{\|}\right)^{2}+x}
$$

and, substituting expansion (22) into equation (20), we find that $B=-A, C=D=0$, i.e., $\varphi\left(p_{\|}\right)=A \gamma_{5}\left(1-i \gamma_{1} \gamma_{2}\right)$, and the function $A$ satisfies the equation

$$
A(p)=\frac{\alpha}{2 \pi^{2}} \int \frac{d^{2} k A(k)}{k^{2}+m_{d y n}^{2}} \int_{0}^{\infty} \frac{d x \exp \left(-x l^{2} / 2\right)}{(\mathbf{k}-\mathbf{p})^{2}+x}
$$

(henceforth we will omit the symbol $\|$ from $p$ and $k$ ). Introducing the function

$$
\Psi(\mathbf{r})=\int \frac{d^{2} k}{(2 \pi)^{2}} \frac{A(k)}{k^{2}+m_{d y n}^{2}} e^{i \mathbf{k r}},
$$

we get (from Eq.(23)) the two-dimensional Schrödinger equation (2) with the potential

$$
\begin{aligned}
V(\mathbf{r}) & =-\frac{\alpha}{2 \pi^{2}} \int d^{2} p e^{i \mathbf{p r}} \int_{0}^{\infty} \frac{d x \exp (-x / 2)}{l^{2} p^{2}+x}=-\frac{\alpha}{\pi l^{2}} \int_{0}^{\infty} d x e^{-x / 2} K_{0}\left(\frac{r}{l} \sqrt{x}\right) \\
& =\frac{\alpha}{\pi l^{2}} \exp \left(\frac{r^{2}}{2 l^{2}}\right) E i\left(-\frac{r^{2}}{2 l^{2}}\right)
\end{aligned}
$$

( $K_{0}$ is the Bessel function). Since $-m_{d y n}^{2}$ plays the role of energy $E$ in this equation and $V(\mathbf{r})$ is a negative, i.e. attractive, potential, the problem is reduced to finding the spectrum of bound states (with $E=-m_{d y n}^{2}<0$ ) of the two-dimensional Schrödinger equation with the potential (24). For this purpose, we can use some results proved in the literature. First, the energy of the lowest level $E(\alpha)$ for the two-dimensional Schrödinger equation is a nonanalytic function of the coupling constant $\alpha$ at $\alpha=0$ [14]. Second, if the potential $V(\mathbf{r})$ were short-range, then $m_{d y n}^{2}(\alpha)=-E(\alpha)$ would take the form $m_{d y n}^{2} \sim \exp [-1 /(a \alpha)]$ where $a$ is a positive constant [14]. However, the potential is long-range in our case. Indeed, using the asymptotic relations for $\operatorname{Ei}(x)$ [5], we get:

$$
\begin{aligned}
& V(\mathbf{r}) \simeq-\frac{2 \alpha}{\pi} \frac{1}{r^{2}}, \quad r \rightarrow \infty ; \\
& V(\mathbf{r}) \simeq-\frac{\alpha}{\pi l^{2}}\left(\gamma+\ln \frac{2 l^{2}}{r^{2}}\right), \quad r \rightarrow 0
\end{aligned}
$$


where $\gamma \simeq 0.577$ is the Euler constant. To find $m_{d y n}^{2}(\alpha)$, we shall use the integral equation (23) at $p=0$. Then, as $\alpha \rightarrow 0$, the dominant contribution in the integral on the right hand side of Eq.(23) is formed in the infrared region with soft $k^{2} \lesssim m_{d y n}^{2}$ :

$$
A(0) \simeq \frac{\alpha}{2 \pi^{2}} A(0) \int \frac{d^{2} k}{k^{2}+m_{d y n}^{2}} \int_{0}^{\infty} \frac{d x \exp (-y / 2)}{l^{2} k^{2}+y} \sim \frac{\alpha}{4 \pi} A(0)\left[\ln \left(\frac{m_{d y n}^{2} l^{2}}{2}\right)\right]^{2}
$$

i.e.,

$$
m_{d y n}=C \sqrt{|e B|} \exp \left[-\sqrt{\frac{\pi}{\alpha}}\right]
$$

where the constant $C=O\left(\alpha^{0}\right)$ remains undefined in this approximation. Note that this result agrees with the analysis of Ref.[15] where the analytic properties of $E(\alpha)$ were considered for the Schrödinger equations with potentials having the asymptotics $V(r) \rightarrow 1 / r^{2}$ as $r \rightarrow \infty$.

Let us now turn to considering the general covariant gauge (8). As is known, the ladder approximation is not gauge invariant. However, let us show that, because the present effect is due to the infrared dynamics in QED, where the coupling constant is small, the leading term in $\ln \left(m_{d y n}\right), \ln \left(m_{d y n}\right) \simeq-(\pi / \alpha)^{1 / 2}$, is the same in all covariant gauges.

Acting in the same way as before, we find that in the general covariant gauge the wave function (22) takes the form $\varphi(p)=\gamma_{5}\left(1-i \gamma_{1} \gamma_{2}\right)(A+\hat{p} C)$ where the functions $A$ and $C$ satisfy the equations:

$$
\begin{gathered}
A(p)=\frac{\alpha}{2 \pi^{2}} \int \frac{d^{2} k A(k)}{k^{2}+m_{d y n}^{2}} \int_{0}^{\infty} \frac{d x\left(1-\lambda x l^{2} / 4\right) \exp \left(-x l^{2} / 2\right)}{(\mathbf{k}-\mathbf{p})^{2}+x} \\
C(p)=\frac{\alpha \lambda}{4 \pi^{2}} \int \frac{d^{2} k C(k)}{k^{2}+m_{d y n}^{2}}\left[2 k^{2}-(\mathbf{k p})-\frac{k^{2}(\mathbf{k p})}{p^{2}}\right] \int_{0}^{\infty} \frac{d x \exp \left(-x l^{2} / 2\right)}{\left[(\mathbf{k}-\mathbf{p})^{2}+x\right]^{2}} .
\end{gathered}
$$

One can see that the dominant contribution on the right-hand side of Eq.(28) (proportional to $\left[\ln m_{d y n}^{2} l^{2}\right]^{2}$ and formed at small $k^{2}$ ) is independent of the gauge parameter $\lambda$. Therefore the leading singularity in $\ln \left(m_{d y n}\right), \ln \left(m_{d y n}\right)=-(\pi / \alpha)^{1 / 2}$, is indeed gauge invariant.

This concludes the derivation of Eqs.(11) and (2) describing spontaneous chiral symmetry breaking by a magnetic field in QED. 
In conclusion, let us discuss possible applications of this effect. One potential application is the interpretation of the results of the GSI heavy-ion scattering experiments [16] in which narrow peaks are seen in the energy spectra of emitted $e^{+} e^{-}$pairs. One proposed explanation [17] is that a very strong electromagnetic field, created by the heavy ions, induces a phase transition in QED to a phase with spontaneous chiral symmetry breaking. The observed peaks are due to the decay of positronium-like states in this phase. The catalysis of chiral symmetry breaking by a magnetic field in QED, studied in this paper, can serve as a toy example of such a phenomenon. In order to get a more realistic model, it would be interesting to extend this analysis to non-constant background fields 18 .

Another, potentialy interesting, application can be connected with the possibility of the existence of very strong magnetic fields ( $B \sim 10^{24}$ Gauss) during the electroweak phase transition in the early Universe [19]. As the present results suggest, such fields might essentially change the character of the electroweak phase transition.

Yet another application of the effect can be connected with the role of iso- and chromomagnetic backgrounds as models for the QCD vacuum (the Copenhagen vacuum [20]). Also, as has been suggested recently [21], isomagnetic fields in the vacuum of electroweak left-right models can induce the parity breakdown. Our work suggests that such field configurations may play the important role in triggering chiral symmetry breaking in QCD and those left-right models.

The work of I.A.Sh. was supported in part by the International Soros Science Education Program (ISSEP) through grant No.PSU052143.

\section{Note added in proof:}

We have just finished a complete (both analytical and numerical) analysis of integral equation (23). The result for $m_{\text {dyn }}$ agrees quite well with estimate (27) and is $m_{\text {dyn }}=$ $C \sqrt{|e B|} \exp \left[-\pi / 2 \sqrt{\pi / 2 \alpha}\right.$, where $C$ is of $O\left(\alpha^{0}\right)$. Notice that the ratio of the powers of this exponent and that in Eq.(27) is $\pi / 2 \sqrt{2} \simeq 1.1$. 


\section{References}

[1] J. Schwinger, Phys. Rev. 82, 664 (1951); Phys. Rev. D7, 1696 (1973).

[2] D.Caldi, A.Chodos, K.Everding, D.Owen, and S.Vafaeisefat, Phys. Rev. D39, 1432 (1989).

[3] V.P.Gusynin, V.A.Miransky and I.A.Shovkovy, Phys. Rev. Lett. 73, 3499 (1994); Phys. Rev. D52, 4718 (1995).

[4] V.P.Gusynin, V.A.Miransky and I.A.Shovkovy, Phys. Lett. B349, 477 (1995).

[5] I.S. Gradshtein and I.M. Ryzhik, Table of Integrals, Series and Products (Academic Press, Orlando, 1980).

[6] P.I.Fomin, V.P.Gusynin, V.A.Miransky, and Yu.A.Sitenko, Riv. del Nuov. Cim. 6 N5 (1983).

[7] V.A. Miransky, Dynamical Symmetry Breaking in Quantum Field Theories (World Scientific Co., Singapore, 1993).

[8] J.E.Avron, I.W.Herbst and B.Simon, Ann. Phys. 114, 431 (1978).

[9] A.I. Akhiezer and V.B. Berestetsky, Quantum Electrodynamics (Interscience, NY, 1965).

[10] A.Chodos, K.Everding and D.A.Owen, Phys. Rev. D42, 2881 (1990).

[11] The Lorentz invariance is broken by a magnetic field in this problem. By the $(3+1)-$ dimensional form, we understand that the denominator of the propagator depends on energy and all the components of momentum: $D(P) \sim\left(P_{0}^{2}-C_{\perp} P_{\perp}^{2}-C_{\|} P_{\|}^{2}\right)^{-1}(D(P) \sim$ $\left(P_{0}-C_{\perp} P_{\perp}^{2}-C_{\|} P_{\|}^{2}\right)^{-1}$ in nonrelativistic systems) with $C_{\perp}, C_{\|} \neq 0$.

[12] B.R. Johnson, J.O. Hirschfelder, K.-H. Yang, Rev. Mod. Phys. 55, 109 (1983). 
[13] N.D. Mermin and H. Wagner, Phys. Rev. Lett. 17 (1966) 1133; S. Coleman, Commun. Math. Phys. 31 (1973) 259.

[14] B. Simon, Ann. Phys. 97, 279 (1976).

[15] A.M.Perelomov and V.S.Popov, Theor. Math. Phys. 4, 664 (1970).

[16] P.Salabura et al., Phys. Lett. B245, 153 (1990); I.Koenig et al., Z. Phys. A346, 153 (1993).

[17] L.S.Celenza, V.K.Mishra, C.M.Shakin and K.F.Lin, Phys. Rev. Lett. 57, 55 (1986); D.G.Caldi and A.Chodos, Phys. Rev. D36, 2876 (1987); Y.J.Ng and Y.Kikuchi, Phys. Rev. D36, 2880 (1987).

[18] D.G.Caldi and S.Vafaeisefat, Phys. Lett. B287, 185 (1992).

[19] T.Vachaspati, Phys. Lett. B265, 258 (1991).

[20] N.K.Nielsen and P.Olesen, Nucl. Phys. B144, 376 (1978).

[21] R.Bönish, DESY report 94-129, hep-th/9407162. 